\title{
Joint Transmitter-Receiver Beamforming in Downlink Cyclic Prefix-free Spatio-Temporal MC-CDMA
}

\author{
Ho Huat Peh*, Athanassios Manikas ${ }^{\dagger}$, Tjeng Thiang Tjhung* and Wai-Choong Wong ${ }^{\ddagger}$ \\ *Institute for Infocomm Research, A*STAR, Singapore \\ Email: $\{$ hhpeh, tjhungtt\}@i2r.a-star.edu.sg \\ $\dagger$ Department of Electrical and Electronic Engineering, Imperial College London, UK \\ Email: a.manikas@imperial.ac.uk \\ ${ }^{\ddagger}$ Department of Electrical and Computer Engineering, National University of Singapore, Singapore \\ Email: elewwcl@nus.edu.sg
}

\begin{abstract}
The problem of jointly optimizing the transmitter and receiver beamforming weights in the downlink of a cyclic prefix-free MC-CDMA system over multipath fading channels is addressed in this paper. Both the base station and user terminals are equipped with antenna arrays to leverage on the spatial information provided by the antenna arrays. The precoding and equalization matrices are designed with the aim of minimizing the overall mean-squared-error (MSE) of the system subject to a power constraint for each transmitter antenna, which has the additional benefit of reducing the probability of clipping of the transmitted signals from the transmitter. This is highly favorable for a MC-CDMA system due to the multicarrier modulation involved. The performance of the optimization process is supported by simulation results.
\end{abstract}

$\begin{array}{ll}a & \text { Scalar. } \\ \underline{a}, \underline{A} & \begin{array}{l}\text { Column vector. } \\ \mathbb{A}\end{array} \\ \mathbb{I}_{N} & \text { Matrix. } \\ \mathbb{O}_{M \times N} & M \times N \text { identity matrix. } \\ \underline{0}_{N} & N \text { element column vector of zeros. } \\ \underline{\iota}_{N, k} & N \times 1 \text { vector with } 1 \text { at the } k^{\text {th }} \text { element and } \\ ()^{H} & \text { Os elsewhere. } \\ ()^{T} & \text { Hermitian transpose. } \\ \otimes & \text { Transpose. } \\ \operatorname{diag}(\underline{a}) & \text { Kronecker product. } \\ \exp (\underline{a}) & \text { Elementwise exponential of vector } \underline{a} . \\ \operatorname{tr}(\mathbb{A}) & \text { Trace of matrix } \mathbb{A} .\end{array}$

\section{INTRODUCTION}

In future communication systems, high data rates are required to support the anticipated multi-media intensive applications. Multiple-Input Multiple-Output (MIMO) systems are an effective strategy to achieve the high data rates required in such applications. This is because MIMO systems take advantage of the spatial diversity that is presented through the use of multiple antennas, and exploit the multipath scattering present in the wireless channel which can be achieved by techniques such as spatial multiplexing, e.g. V-BLAST [1] and space-time coding [2]. In addition, multi-carrier (MC) modulation techniques such as orthogonal frequency division multiplexing (OFDM) and MC code division multiple access (MC-CDMA) [3], are expected to be implemented in future MIMO systems, such as MIMO MC-CDMA systems, due to their ability to overcome the detrimental effects of the wireless channel such as multipath effects and frequencyselective fading.

In downlink MIMO systems, there has been considerable interest in the optimization of the transmitter and receivers in multiple access systems. In [4], a variety of design criteria were unified in a generalized framework for the joint optimization process of a MC-MIMO system. In MC-based systems, the peak-to-average power ratio (PAPR) is an important area of concern as the peak transmit power, often limited by regulatory or application restrictions [5], would have to be clipped when the threshold is exceeded. It was shown in [4] that the probability of clipping of an OFDM signal can be reduced through the imposition of a constraint on the transmitted power.

This paper focuses on the downlink of a cyclic prefix-free MC-CDMA system, employing antenna arrays at both the transmitter and receivers, over a frequency-selective fading channel. The use of antenna arrays enables the geometrical information of the antennas and channel to be leveraged upon as array signal processing techniques can be used. This is in contrast to the systems in [6], [7] which ignore the antenna array geometry and so, do not have access to the geometrical information of the channel for improved signal processing capabilities. Furthermore, due to the MC nature of the system, this paper seeks to reduce the probability of clipping of the transmitted signals from each antenna by making use of the relation between the probability of clipping and the average transmit signal power of each antenna. The joint optimization of the precoding and equalization matrices is thus performed with the goal of minimizing the overall MSE of the system, subject to a constraint on the transmitted power of each antenna element. Moreover, instead of the subcarrier- 
Base Station Modulation and Transmit Beamforming

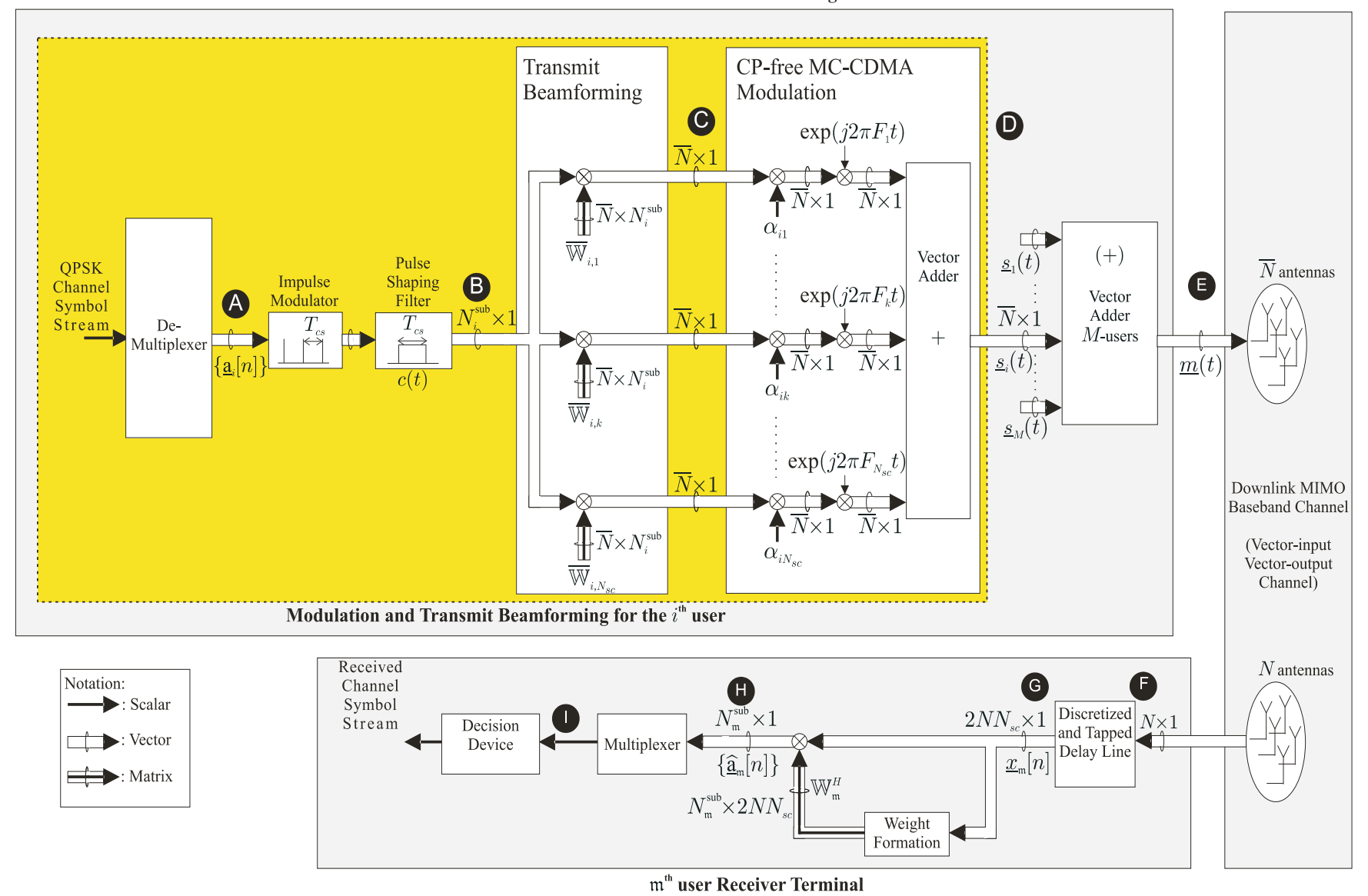

Fig. 1. System architecture of the downlink of a multi-user cyclic prefix-free MC-CDMA arrayed MIMO communication system.

noncooperative approach, the subcarrier-cooperative approach is used here which implies that the subcarriers are not required to be orthogonal. Thus, there can be flexibility in the allocation of channel symbols among the subcarriers and transmitter antennas to overcome channel fading effects [4].

In Section II, the system and channel models are developed and explained for a downlink MIMO MC-CDMA. The joint optimization process is then developed in Section III and in Section IV, simulation results based on the proposed algorithm are presented. Finally, the paper is concluded in Section V.

\section{System Modelling}

The antenna array-based downlink MIMO MC-CDMA system is shown in Fig. 1. The base station transmitter is equipped with an antenna array of $\bar{N}$ elements, while each receiver terminal has an $N$ element antenna array. Note that symbols with a 'bar' at the top is associated with the transmitter. The channel symbol (e.g. QPSK) stream of the $i^{\text {th }}$ user is demultipexed into $N_{i}^{\text {sub }}$ streams, where $N_{i}^{\text {sub }}$ is the number of substreams, to form the $n^{\text {th }}$ channel symbol vector $\underline{\mathrm{a}}_{i}[n]=\left[\mathrm{a}_{i 1}[n], \mathrm{a}_{i 2}[n], \ldots, \mathrm{a}_{i N_{i}^{\text {sub }}}[n]\right]^{T}$. The vector-symbol sequence $\left\{\underline{\mathrm{a}}_{i}[n], \forall n\right\}$ at point- $\mathrm{A}$ is then converted into the vector-signal at point- $\mathrm{B}$, defined as:

$$
\sum_{n=-\infty}^{n=+\infty} \underline{\mathrm{a}}_{i}[n] c\left(t-n T_{c s}\right), \quad n T_{c s} \leq t<(n+1) T_{c s}
$$

where $c(t)$ is the rectangular pulse of duration $T_{c s}$. The vectorsignal is first copied into $N_{s c}$ parallel streams and the $k^{\text {th }}$ copy of the signal given by (1) then undergoes transmit beamforming through the application of the transmit beamforming matrix of the $i^{\text {th }}$ user at the $k^{\text {th }}$ subcarrier, $\overline{\mathbb{W}}_{i, k} \in \mathcal{C}^{\bar{N} \times N_{i}^{\text {sub }}}$, to obtain the signal at point-C of Fig. 1. Cyclic prefix-free MC-CDMA modulation is then performed by multiplying the $k^{\text {th }}$ branch with a corresponding $k^{\text {th }}$ chip $\alpha_{i k}= \pm 1$ of $\underline{\alpha}_{i}=\left[\alpha_{i 1}, \ldots, \alpha_{i k}, \ldots, \alpha_{i N_{s c}}\right]^{T}$, which is the PN sequence of length $N_{s c}$ corresponding to the $i^{\text {th }}$ user. The resultant vector is then applied to a subcarrier with frequency given by $F_{k}=k \triangle F$, where $\triangle F=1 / T_{c s}$ is the subcarrier separation. The modulated subcarriers are then summed up to produce the baseband MC-CDMA vector-signal $\underline{s}_{i}(t) \in \mathcal{C}^{\bar{N} \times 1}$ :

$$
\begin{aligned}
\underline{s}_{i}(t)= & \sum_{n=-\infty}^{n=+\infty} \underline{\mathrm{a}}_{i}[n] \sum_{k=1}^{N_{s c}} \alpha_{i k} \overline{\mathbb{W}}_{i, k} \exp \left(j 2 \pi F_{k} t\right) \\
& \text { with } n T_{c s} \leq t<(n+1) T_{c s}
\end{aligned}
$$

For $M$ users, the transmitted signal is given by 


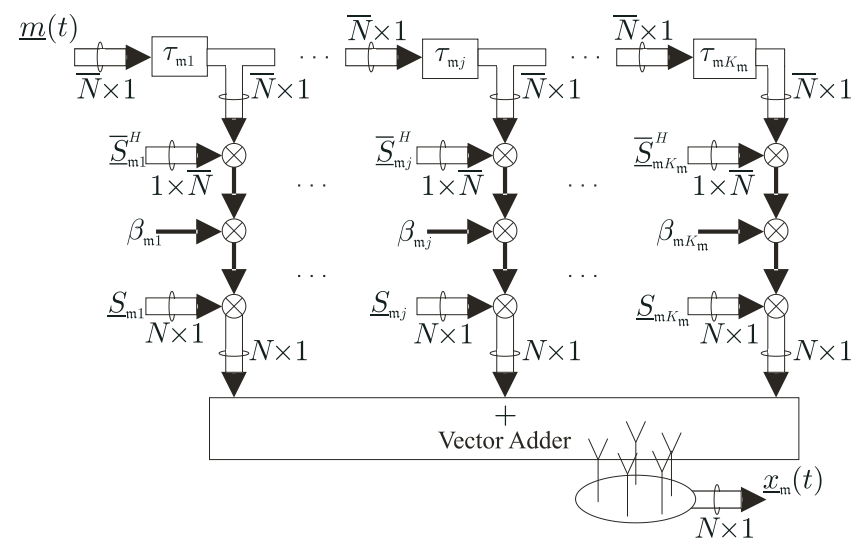

Fig. 2. Vector-signal-input vector-signal-output (VIVO) channel for downlink MIMO system.

$$
\underline{m}(t)=\sum_{i=1}^{M} \underline{s}_{i}(t)
$$

The baseband signal $\underline{s}_{i}(t)$ is upconverted, using a carrier frequency $F_{c}$, to a bandpass signal. However, in this paper, without any loss of generality, the carrier will be ignored and baseband transmission will be assumed.

Based on (3), the total transmitted power of $M$ users is given by $\bar{P}=\sum_{i=1}^{M} \operatorname{tr}\left(\overline{\mathbb{W}}_{i} \overline{\mathbb{W}}_{i}^{H}\right)$, where

$$
\overline{\mathbb{W}}_{i}=\left[\overline{\mathbb{W}}_{i, 1}^{T}, \ldots, \overline{\mathbb{W}}_{i, k}^{T}, \ldots, \overline{\mathbb{W}}_{i, N_{s c}}^{T}\right]^{T}
$$

Also, the total transmitted power from $p^{\text {th }}$ transmit antenna can be expressed as $\bar{P}_{p}=\sum_{i=1}^{M} \operatorname{tr}\left(\overline{\mathbb{W}}_{i} \overline{\mathbb{W}}_{i}^{H}\left(\mathbb{I}_{N_{s c}} \otimes \underline{\iota} \underline{N}_{, p} \underline{\underline{\imath}} \bar{N}, p\right)\right)$.

The wireless channel of the array-based MIMO MC-CDMA system can be modelled as a vector-signal-input vector-signaloutput (VIVO) channel which is assumed to be multipath dispersive with a delay spread of $T_{c s}$. The transmitter and receiver antenna arrays are assumed to have small apertures which implies that each subcarrier present in the signals leaving the transmitter antenna array experiences a common fading channel towards the receiver antenna array elements. Thus, the channel is modelled as a function of the array manifold vectors of both the transmitter and the receiver, as shown in Fig. 2.

The array manifold vector $\underline{S}_{\mathfrak{m} j k}$ of a receive antenna array is dependent on the array geometry, direction-of-arrival (DOA) with azimuth $\theta_{\mathfrak{m} j}$ and elevation $\phi_{\mathfrak{m} j}$ and frequency $\left(F_{c}+F_{k}\right)$ of the received signal, as given by

$$
\underline{S}_{\mathfrak{m} j k}=\exp \left(\frac{-j 2 \pi\left(F_{c}+F_{k}\right)}{c}\left[\underline{r}_{1}, \underline{r}_{2}, \ldots, \underline{r}_{N}\right]^{T} \underline{u}_{j}\right)
$$

where $\underline{u}_{j}=\left[\cos \left(\theta_{j}\right) \cos \left(\phi_{j}\right), \sin \left(\theta_{j}\right) \cos \left(\phi_{j}\right), \sin \left(\phi_{j}\right)\right]^{T}$ is a unit vector in the direction $\left(\theta_{j}, \phi_{j}\right), c$ is the speed of light and the vector $\underline{r}_{i}, i \in 1, \ldots, N$, denotes the Cartesian coordinates of the $i^{\text {th }}$ element of the receive antenna array.
A similar expression can be also written for the array manifold of the transmit antenna array, $\underline{S}_{\mathfrak{m} j k}$, as a function of the direction-of-departure (DOD) $\left(\bar{\theta}_{\mathfrak{m} j}, \bar{\phi}_{\mathfrak{m} j}\right)$. However, when $\max _{k}\left(\frac{k \triangle F}{F_{c}}\right) \rightarrow 0$, the transmit and receive array manifold vectors can be assumed to be independent of the subcarrier frequency, i.e. $F_{c}+F_{k} \simeq F_{c}$. Thus, as in Fig. 2, the dependence of the array manifold vectors on the subcarrier frequency has been removed. In Fig. 2, $\tau_{\mathfrak{m} j}$ and $\beta_{\mathfrak{m} j}$ represents the path delay and fading coefficient of the $j^{\text {th }}$ path with $j \in 1,2, \ldots, K_{\mathfrak{m}}$ and $K_{\mathfrak{m}}$ is the number of resolvable multipaths from the base station to the $\mathfrak{m}^{\text {th }}$ user. The baseband received signal $\underline{x}_{\mathfrak{m}}(t)$ at the $\mathfrak{m}^{\text {th }}$ user can be written as

$$
\underline{x}_{\mathfrak{m}}(t)=\sum_{j=1}^{K_{\mathfrak{m}}} \beta_{\mathfrak{m} j} \underline{S}_{\mathfrak{m} j} \underline{S}_{\mathfrak{m} j \underline{m}}^{H}\left(t-\tau_{\mathfrak{m} j}\right)+\underline{\mathfrak{n}}_{\mathfrak{m}}(t)
$$

where $\underline{\mathrm{n}}_{\mathfrak{m}}(t)$ denotes the Additive White Gaussian Noise (AWGN) of power $\sigma_{\mathfrak{m}}^{2}$ present in the channel.

With reference to Fig. 1, the signal at point-F from each receive antenna is sampled with a rate of $\frac{1}{T_{s}}$, where $T_{s}=\frac{T_{c s}}{N_{s c}}$. Hence, $N_{s c}$ samples are obtained per channel symbol period and a tapped delay line of length $2 N_{s c}$ (equivalent to $2 T_{c s}$ ) applied at each antenna enables the asynchronous receiver to capture the contribution of a full symbol (current symbol). A vector of length $2 N N_{s c}, \underline{x}[n]$, is then formed at point-G of Fig. 1 by concatenating the contents of the tapped delay lines, read at a rate of $\frac{1}{T_{c s}}$. A subcarrier vector, $\underline{\mathrm{f}}_{k}[\ell]$, given by

$$
\underline{\mathrm{f}}_{k}[\ell]=\left[\begin{array}{c}
\exp \left(j 2 \pi F_{k}(-\ell) T_{s}\right) \\
\exp \left(j 2 \pi F_{k}(1-\ell) T_{s}\right) \\
\vdots \\
\exp \left(j 2 \pi F_{k}\left(N_{s c}-1-\ell\right) T_{s}\right)
\end{array}\right]
$$

models the time variation of the $k^{\text {th }}$ subcarrier after undergoing a path delay of $\ell$ sample periods where $\ell \in 0,1, \ldots,\left(N_{s c}-1\right)$. Thus, (6) can be rewritten as shown in (8).

$$
\begin{aligned}
& \underline{x}_{\mathfrak{m}}[n]=\sum_{i=1}^{M} \sum_{j=1}^{K_{\mathfrak{m}}} \sum_{k=1}^{N_{s c}} \beta_{\mathfrak{m} j} \underline{S}_{\mathfrak{m} j} \otimes \\
& \left(\begin{array}{c}
\alpha_{i k}\left(\mathbb{J}^{T}\right)^{N_{s c}} \mathbb{J}^{\ell_{\mathfrak{m} j}}\left[\begin{array}{c}
\underline{\mathrm{f}}_{k}\left[\ell_{\mathfrak{m} j}\right] \\
\underline{0}_{N_{s c}}
\end{array}\right] \underline{\underline{S}}_{\mathfrak{m} j}^{H} \overline{\mathbb{W}}_{i, k} \underline{\mathrm{a}}_{i}[n-1] \\
+\alpha_{i k} \mathbb{J}^{\ell_{\mathfrak{m} j}}\left[\begin{array}{c}
\underline{\mathrm{f}}_{k}\left[\ell_{\mathfrak{m} j}\right] \\
\underline{0}_{N_{s c}}
\end{array}\right] \underline{S}_{\mathfrak{m} j}^{H} \overline{\mathbb{W}}_{i, k} \underline{\mathrm{a}}_{i}[n] \\
+\alpha_{i k}(\mathbb{J})^{N_{s c}} \mathbb{J}^{\ell_{\mathfrak{m} j}}\left[\begin{array}{c}
\underline{\mathrm{f}}_{k}\left[\ell_{\mathfrak{m} j}\right] \\
\underline{0}_{N_{s c}}
\end{array}\right] \underline{\bar{S}}_{\mathfrak{m} j}^{H} \overline{\mathbb{W}}_{i, k} \underline{\mathbf{a}}_{i}[n+1]
\end{array}\right) \\
& +\underline{\mathrm{n}}_{m}[n]
\end{aligned}
$$

The matrix $\mathbb{J}\left(\right.$ or $\mathbb{J}^{T}$ ) is a $2 N_{s c} \times 2 N_{s c}$ time down-shift (or up-shift) operator defined by

$$
\mathbb{J}=\left[\begin{array}{lc}
\underline{0}_{2 N_{s c}-1}^{T} & 0 \\
\mathbb{I}_{2 N_{s c}-1} & \underline{0}_{2 N_{s c}-1}
\end{array}\right]
$$

and it is used to model the delay $\ell$ when $\mathbb{J}^{\ell}$ or $\left(\mathbb{J}^{T}\right)^{\ell}$ is applied on the column vector $\left[\underline{\mathrm{f}}_{k}^{T}[\ell], \quad \underline{0}_{N_{s c}}^{T}\right]^{T}$.

A multi-carrier space-time array (MC-STAR) manifold ma- 
trix for the $j^{\text {th }}$ path of $i^{\text {th }}$ user, as observed at the $\mathfrak{m}^{\text {th }}$ user, is defined as

$$
\mathbb{H}_{\mathfrak{m}, i j}=\underline{S}_{\mathfrak{m} j} \otimes\left(J^{\ell_{\mathfrak{m} j}}\left[\begin{array}{c}
\mathbb{F}_{\ell_{\mathfrak{m} j}} \operatorname{diag}\left(\underline{\alpha}_{i}\right) \\
\mathbb{O}_{N_{s c} \times N_{s c}}
\end{array}\right]\left(\mathbb{I}_{N_{s c}} \otimes \underline{S}_{\mathfrak{m} j}^{H}\right)\right)
$$

which is a function of the receive and transmit array manifold vectors, $\underline{S}_{\mathfrak{m} j}$ and $\underline{\bar{S}}_{\mathfrak{m} j}$, respectively, and the path delay $\ell_{\mathfrak{m} j}$ for the $j^{\text {th }}$ path as observed at the $\mathfrak{m}^{\text {th }}$ user as well as the PN sequence of the $i^{\text {th }}$ user and

$$
\mathbb{F}_{\ell_{\mathfrak{m} j}}=\left[\underline{\mathrm{f}}_{1}\left[\ell_{\mathfrak{m} j}\right], \ldots, \underline{\mathrm{f}}_{k}\left[\ell_{\mathfrak{m} j}\right], \ldots, \underline{\mathrm{f}}_{N_{s c}}\left[\ell_{\mathfrak{m} j}\right]\right]
$$

Thus, $\mathbb{H}_{\mathfrak{m}, i j}$ encompasses the spatial-temporal channel characteristics (DOA and TOA) for the received signal at the $\mathfrak{m}^{\text {th }}$ user and blind channel estimation algorithms such as that in [8] can be used to obtain estimates of the spatial-temporal channel parameters. Thus, (8) can be expressed as

$$
\underline{x}_{\mathfrak{m}}[n]=\sum_{i=1}^{M}\left(\mathbb{V}_{\mathfrak{m}, i}\left(\mathbb{I}_{3} \otimes \overline{\mathbb{W}}_{i}\right)\left[\begin{array}{c}
\underline{\mathrm{a}}_{i}[n-1] \\
\underline{\mathrm{a}}_{i}[n] \\
\underline{\mathrm{a}}_{i}[n+1]
\end{array}\right]\right)+\underline{\mathrm{n}}_{\mathfrak{m}}[n]
$$

where $\mathbb{V}_{\mathfrak{m}, i}=\left[\mathbb{H}_{\mathfrak{m}, i}^{\text {prev }} \mathbb{H}_{\mathfrak{m}, i} \mathbb{H}_{\mathfrak{m}, i}^{\text {next }}\right]$ and $\mathbb{H}_{\mathfrak{m}, i}, \mathbb{H}_{\mathfrak{m}, i}^{\text {prev }}$ and $\mathbb{H}_{\mathfrak{m}, i}^{\text {next }}$ are defined as

$$
\mathbb{H}_{\mathfrak{m}, i}=\sum_{j=1}^{K_{\mathfrak{m}}} \beta_{\mathfrak{m} j} \mathbb{H}_{\mathfrak{m}, i j}
$$

$\mathbb{H}_{\mathfrak{m}, i}^{\text {prev }}=\left(\mathbb{I}_{N} \otimes\left(\mathbb{J}^{T}\right)^{N_{s c}}\right) \mathbb{H}_{\mathfrak{m}, i} ; \mathbb{H}_{\mathfrak{m}, i}^{\text {next }}=\left(\mathbb{I}_{N} \otimes \mathbb{J}^{N_{s c}}\right) \mathbb{H}_{\mathfrak{m}, i}$

In the $\mathfrak{m}^{\text {th }}$ receiver, the receive beamforming weight, $\mathbb{W}_{\mathfrak{m}}^{H}$, is used to obtain the soft decision variable vector, $\widehat{\widehat{a}}_{\mathfrak{m}}[n]$, which is given by

$$
\underline{\widehat{a}}_{\mathfrak{m}}[n]=\mathbb{W}_{\mathfrak{m}}^{H} \underline{x}_{\mathfrak{m}}[n]
$$

\section{JOINT TRANSMIT-RECEIVE BEAMFORMER OPTIMIZATION}

The objective of the optimization process is to determine the precoder and equalization matrices, $\overline{\mathbb{W}}_{\mathfrak{m}}$ and $\mathbb{W}_{\mathfrak{m}}$, respectively, for each user with a predetermined number of substreams for each user. Thus, based on the soft decision variable vector, $\underline{\widehat{a}}_{\mathfrak{m}}[n]$ in (13), the MSE matrix of the $\mathfrak{m}^{\text {th }}$ receiver can be expressed as

$$
\begin{aligned}
\mathbb{E}_{\mathfrak{m}} & =\mathcal{E}\left\{\left(\underline{\widehat{a}}_{\mathfrak{m}}[n]-\underline{\mathrm{a}}_{\mathfrak{m}}[n]\right)\left(\underline{\widehat{a}}_{\mathfrak{m}}[n]-\underline{\mathrm{a}}_{\mathfrak{m}}[n]\right)^{H}\right\} \\
& =\mathbb{W}_{\mathfrak{m}}^{H} \mathbb{R}_{x x, \mathfrak{m}} \mathbb{W}_{\mathfrak{m}}+\mathbb{I}_{N_{\mathfrak{m}}^{s u b}} \\
& -\mathbb{W}_{\mathfrak{m}}^{H} \mathbb{H}_{\mathfrak{m}, \mathfrak{m}} \overline{\mathbb{W}}_{\mathfrak{m}}-\overline{\mathbb{W}}_{\mathfrak{m}}^{H} \mathbb{H}_{\mathfrak{m}, \mathfrak{m}}^{H} \mathbb{W}_{\mathfrak{m}}
\end{aligned}
$$

where

$$
\begin{aligned}
\mathbb{R}_{x x, \mathfrak{m}} & =\mathcal{E}\left\{\underline{x}_{\mathfrak{m}}[n] \underline{x}_{\mathfrak{m}}^{H}[n]\right\} \\
& =\sum_{i=1}^{M} \mathbb{V}_{\mathfrak{m}, i}\left(\mathbb{I}_{3} \otimes \overline{\mathbb{W}}_{i} \overline{\mathbb{W}}_{i}^{H}\right) \mathbb{V}_{\mathfrak{m}, i}^{H}+\sigma_{\mathfrak{m}}^{2} \mathbb{I}_{2 N N_{s c}}
\end{aligned}
$$

denotes the received signal covariance matrix of the $\mathfrak{m}^{\text {th }}$ receiver. The optimization objective of minimizing the MSE of all users can thus be expressed as the following constraint optimization problem

$$
\begin{gathered}
\min _{\left(\overline{\mathbb{W}}_{1}, \ldots, \overline{\mathbb{W}}_{M} ; \mathbb{W}_{1}, \ldots, \mathbb{W}_{M}\right)} \sum_{\mathfrak{m}=1}^{M} \operatorname{tr}\left(\mathbb{E}_{\mathfrak{m}}\right) \\
\text { s.t. } \sum_{\mathfrak{m}=1}^{M} \operatorname{tr}\left(\overline{\mathbb{W}}_{\mathfrak{m}} \overline{\mathbb{W}}_{\mathfrak{m}}^{H}\left(\mathbb{I}_{N_{s c}} \otimes \underline{\imath} \bar{N}, p-\frac{\underline{T}}{N}, p\right)\right) \leq \bar{P}_{p}, \\
\text { with } p=1, \ldots, \bar{N}
\end{gathered}
$$

where

$$
\bar{P}_{p}=\frac{A_{c l i p}^{2}}{\ln \left(\frac{1}{1-(1-\mathcal{P})^{\frac{1}{L}}}\right)}
$$

and $\operatorname{Pr}\left\{\left|m_{p}(t)\right|>A_{\text {clip }}\right\}=\mathcal{P}$ denotes the probability that the amplitude of a sample of the transmitted signal of the $p^{\text {th }}$ antenna, given by $m_{p}(t)$, is greater than the clipping amplitude, $A_{\text {clip }}$. In obtaining (17), it is assumed that there are $L$ samples in the transmitted MC-CDMA signal and due to the assumption of a Rayleigh distribution for $\left|m_{p}(t)\right|$ [9], the above expression is obtained for the constraint $\bar{P}_{p}$ instead of the expression in [4] which is based on a Gaussian assumption.

The optimization problem stated in (16) shows that the constraint involves limiting the total transmitted power from each transmitter antenna and this is in contrast to [10] which imposes a constraint on the total transmit power for a DSCDMA system. In the following, it is assumed that both the transmitter and receivers have perfect channel knowledge and so, by using the Lagrange Multiplier method, the cost function for the optimization problem can be expressed as:

$$
\begin{aligned}
J & =\sum_{\mathfrak{m}=1}^{M} \operatorname{tr}\left(\mathbb{E}_{\mathfrak{m}}\right) \\
& +\sum_{p=1}^{\bar{N}} \mu_{p}\left(\sum_{\mathfrak{m}=1}^{M} \operatorname{tr}\left(\overline{\mathbb{W}}_{\mathfrak{m}} \overline{\mathbb{W}}_{\mathfrak{m}}^{H}\left(\mathbb{I}_{N_{s c}} \otimes \underline{\iota}_{\bar{N}, p} \frac{\underline{L}}{N, p}\right)-\bar{P}_{p}\right)\right)
\end{aligned}
$$

In order to obtain the solution for the precoding matrix $\overline{\mathbb{W}}_{\mathfrak{m}}$ of the $\mathfrak{m}^{\text {th }}$ receiver, it is assumed that the receive decoding matrix $\mathbb{W}_{i}, \forall i$ are fixed and thus, by evaluating $\left(\frac{\partial J}{\partial \mathbb{W}_{\mathfrak{m}}}\right)_{\overline{\mathbb{W}}_{\mathfrak{m}}=\overline{\mathbb{W}}_{\mathfrak{m}}^{*}}=0$, the solution $\overline{\mathbb{W}}_{\mathfrak{m}}^{*}$ is then given by

$$
\begin{aligned}
\overline{\mathbb{W}}_{\mathfrak{m}}^{*} & =\left\{\mathbb{L}^{H}\left(\sum_{i=1}^{M}\left(\mathbb{V}_{\mathfrak{m}, i}^{H}\left(\mathbb{W}_{i} \mathbb{W}_{i}^{H}\right) \mathbb{V}_{\mathfrak{m}, i}\right)\right) \mathbb{L}\right. \\
& \left.+\sum_{p=1}^{\bar{N}} \mu_{p}\left(\mathbb{I}_{N_{s c}} \otimes \underline{\iota}_{\bar{N}, p} \underline{\underline{L}}_{\bar{N}, p}\right)\right\}^{-1} \mathbb{H}_{\mathfrak{m}, \mathfrak{m}}^{H} \mathbb{W}_{\mathfrak{m}}
\end{aligned}
$$

where $\mathbb{L}=\underline{1}_{3} \otimes \mathbb{I}_{\bar{N}} N_{s c}$.

Also, the solution for the Lagrange Multiplier for the $p^{\text {th }}$ antenna, $\mu_{p}^{*}$, is given by 


$$
\begin{aligned}
\mu_{p}^{*} & =\frac{1}{\bar{P}_{p}} \sum_{\mathfrak{m}=1}^{M} \operatorname{tr}\left\{( \mathbb { I } _ { N _ { s c } } \otimes \underline { \underline { \iota } } \overline { N } , p _ { p } \frac { \underline { T } , p } { N , p } ) \left(\mathbb{H}_{\mathfrak{m}, \mathfrak{m}}^{H} \mathbb{W}_{\mathfrak{m}} \overline{\mathbb{W}}_{\mathfrak{m}}^{H}\right.\right. \\
& \left.\left.-\mathbb{L}^{H}\left(\sum_{i=1}^{M} \mathbb{V}_{\mathfrak{m}, i}^{H}\left(\mathbb{W}_{i} \mathbb{W}_{i}^{H}\right) \mathbb{V}_{\mathfrak{m}, i}\right) \mathbb{L} \overline{\mathbb{W}}_{\mathfrak{m}} \overline{\mathbb{W}}_{\mathfrak{m}}^{H}\right)\right\}
\end{aligned}
$$

Conversely, the precoding matrices are fixed in order to obtain the optimal equalization matrices, which can be expressed as

$$
\mathbb{W}_{\mathfrak{m}}^{*}=\mathbb{R}_{x x, \mathfrak{m}}^{-1} \mathbb{H}_{\mathfrak{m}, \mathfrak{m}} \overline{\mathbb{W}}_{\mathfrak{m}}
$$

corresponding to the MMSE receiver.

Based on the derivations for the precoder and equalization matrices as well as the Lagrangian Multipliers, an iterative algorithm is an attractive option since the precoder and equalization matrices as well as the Lagrangian Multipliers are dependent on each other. Thus, the joint optimization process can be performed iteratively.

1. Initialization: (iterative index $\mathfrak{i}=0$ ) the precoder matrix for each user, $\overline{\mathbb{W}}_{\mathfrak{m}}$, is first initialized as

$$
\overline{\mathbb{W}}_{\mathfrak{m}}^{(i=0)}=\sqrt{\frac{\bar{P}}{M N_{\mathfrak{m}}^{\text {sub }}}} \mathbb{U}_{\mathfrak{m}}^{N_{\mathfrak{m}}^{\text {sub }}}
$$

where the columns of $\mathbb{U}_{\mathfrak{m}}^{N_{\mathrm{m}}^{\text {sub }}}$ are the general eigenvectors corresponding to the $N_{m}^{\text {sub }}$ largest general eigenvectors of the matrix $\mathbb{H}_{\mathfrak{m}, \mathfrak{m}}^{H} \mathbb{H}_{\mathfrak{m}, \mathfrak{m}}$. The coefficient $\sqrt{\frac{\bar{P}}{M N_{\mathfrak{m}}^{\text {sub }}}}$ is required to ensure an equal distribution of power to each user and substream.

2. The equalization matrix $\mathbb{W}_{\mathfrak{m}}^{(\mathfrak{i})}$ is then calculated for each user $\mathfrak{m}=1, \ldots M$ based on (21).

3. Using the $i^{\text {th }}$ precoder and equalization matrices, the Lagrangian multipliers $\mu_{p}^{(i)}, p=1, \ldots \bar{N}$ are then calculated using (20).

4. The equalization matrices and Lagrangian multipliers obtained in the previous 2 steps are used to update the precoder matrices $\overline{\mathbb{W}}_{\mathfrak{m}}^{(i+1)}$ by using (19).

5. $\mathfrak{i}$ is replaced by $\mathfrak{i}+1$ and Steps $2-4$ are repeated until a convergence criterion is satisfied.

\section{Simulation Results}

The performance of the proposed iterative joint optimization algorithm is investigated in this section. The transmitter is assumed to be equipped with an uniform linear array (ULA) of $\bar{N}=4$ elements while the receivers are each equipped with a ULA of $N=2$ elements, all with half-wavelength spacing. Each user is assigned a Gold sequence and $N_{i}^{\text {sub }}=2 \forall i$. It is also assumed that the transmitted signal arrives at each receiver via $K_{i}=4$ multipaths. The DOD and DOA associated with each path are assumed to be uniformly distributed over $\left[0,180^{\circ}\right)$ while the path delay is assumed to be uniformly distributed over $\left[0,\left(N_{s c}-1\right) T_{s}\right]$. A block of 250 channel symbols is collected for processing at each time, during which the channel is assumed to be stationary and both the transmitter and receivers have access to the channel state information.

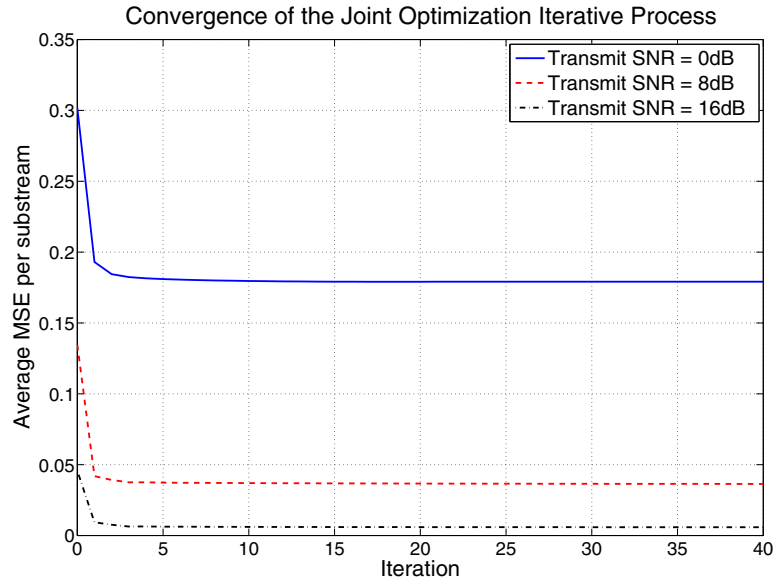

Fig. 3. Study of convergence of the proposed algorithm with transmit SNR = $0,8,16 \mathrm{~dB}$ in a $(6,4,2)$ cyclic prefix-free MC-CDMA arrayed MIMO system $\left(N_{s c}=15\right)$.

The convergence behavior of the proposed iterative algorithm is first studied for a cyclic prefix-free MC-CDMA arrayed MIMO system with $M=6$ users, hereafter known as $(6,4,2)$, i.e. $(M, \bar{N}, N)$ cyclic prefix-free MC-CDMA arrayed MIMO system. The convergence behavior is investigated for different transmit SNR values, defined as $\frac{\bar{P}}{\sigma^{2}}$. Fig. 3 shows the average MSE per substream per user as a function of the iterative process. It can be seen that the convergence property of the proposed iterative algorithm improves with the transmit SNR.

In Fig. 4, the BER performance of the iterative joint optimization algorithm is compared with the transmit precoding scheme described in Step 1 of the proposed algorithm coupled with equalization matrices based on RAKE and MMSE receivers for a $(6,4,2)$ MIMO MC-CDMA system while Fig. 5 shows the BER performance for a $(10,4,2)$ MIMO MC-CDMA system. The precoder and decoder matrices are obtained from 30 iterations. In Figs. 4, and 5, the effect of frequency diversity is also investigated by considering the use of Gold sequences of lengths 7 and 15 in the simulations. As shown in the figures, the iterative process results in a much better BER performance compared to the case where the precoder and decoder matrices are not obtained jointly. This is because of the adaptation of the precoder and decoder matrices, according to the system conditions, during the optimization process. In addition, it has been shown that with frequency diversity, the BER performance of the proposed iterative approach can be improved.

The probability of clipping of the transmitted MC-CDMA signal for various transmit SNR for a $(6,4,2)$ cyclic prefix-free MC-CDMA arrayed MIMO system with $N_{s c}=15$ is next shown in Fig. 6. It is assumed that the transmitted signal is oversampled by a factor of 4 so that the sampled transmitted signal can approximate the continuous time signal. It can be seen that the iterative process results in a lower probability of 


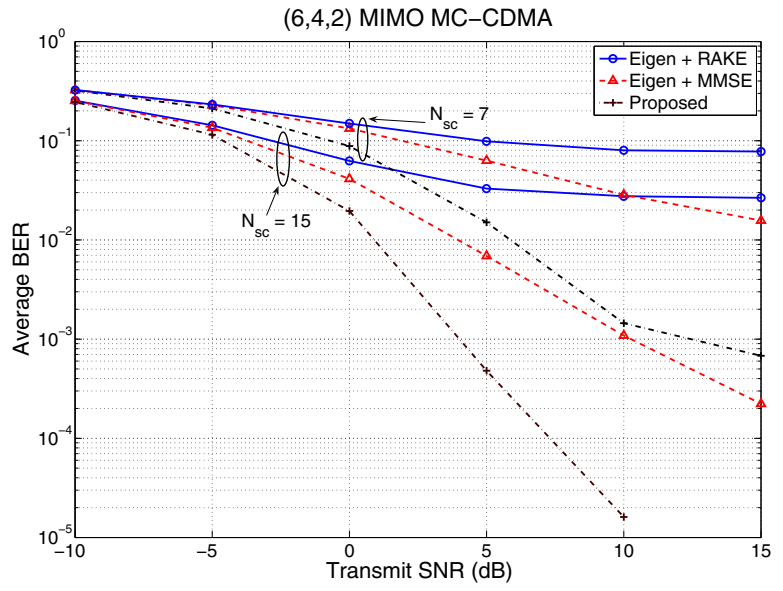

Fig. 4. BER performance of the the proposed iterative algorithm and the eigendecomposition-based transmit precoding with RAKE or MMSE receiver equalization for a $(6,4,2)$ cyclic prefix-free MC-CDMA arrayed MIMO system.

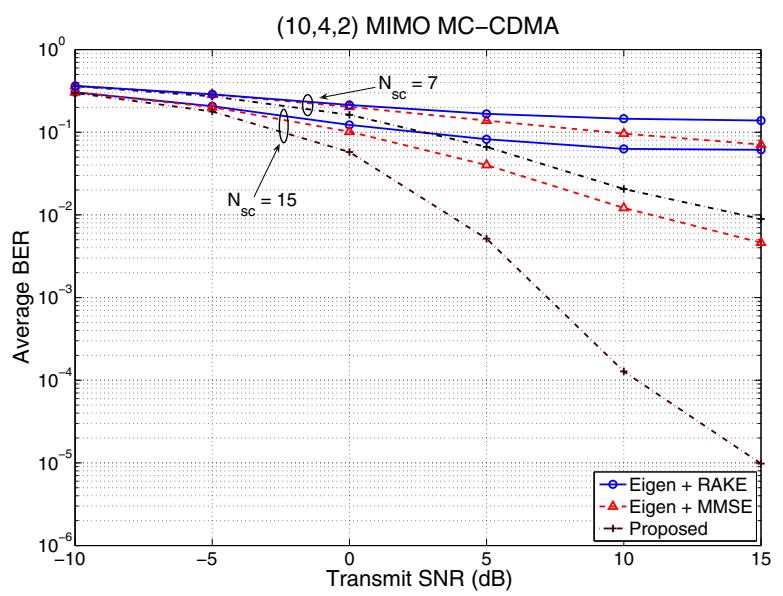

Fig. 5. BER performance of the the proposed iterative algorithm and the eigendecomposition-based transmit precoding with RAKE or MMSE receiver equalization for a $(10,4,2)$ cyclic prefix-free MC-CDMA arrayed MIMO system.

clipping of the transmitted signal compared to the non-iterative case.

\section{CONClusion}

The problem of joint optimization of precoder and equalization matrices in a downlink MIMO MC-CDMA system over multipath fading channels has been investigated in this paper. A subcarrier-cooperative approach has been taken in the optimization of the MC precoding matrices which is a departure from conventional methods where the orthogonality of the subcarriers is required. The proposed approach is based on minimizing the overall MSE with a per-antenna power constraint which can also reduce the probability of clipping of the transmitted MC-CDMA signals. An iterative solution has been provided and simulations have been carried out which

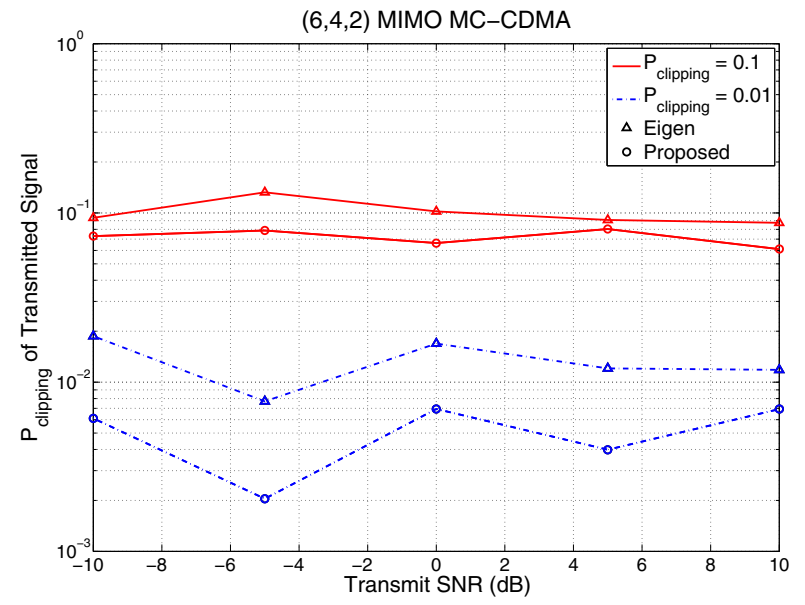

Fig. 6. Average probability of clipping of transmitted MC-CDMA signal from each transmitter antenna with target $\mathrm{P}_{\text {clipping }}=0.1$ and 0.01 for various transmit SNR $\left(N_{s c}=7\right)$.

show the validity of the solution. Perfect channel knowledge is required for the proposed algorithm while the impact of imperfect CSI on the performance of the algorithm will be addressed in future works.

\section{REFERENCES}

[1] P. Wolniansky, G. Foschini, G. Golden, and R. Valenzuela, "V-blast: an architecture for realizing very high data rates over the rich-scattering wireless channel," 1998 URSI International Symposium on Signals, Systems, and Electronics, 1998. ISSSE 98., pp. 295-300, 29 Sep-2 Oct 1998.

[2] S. M. Alamouti, "A simple transmit diversity technique for wireless communications," IEEE Journal on Selected Areas in Communications, vol. 16, no. 8, pp. 1451-1458, October 1998.

[3] N. Yee, J.-P. Linnartz, and G. Fettweis, "Multicarrier CDMA in Indoor Wireless Radio Networks," in 2nd IEEE International Symposium on Personal, Indoor and Mobile Radio Communications, 1993. PIMRC 1993., vol. 1, September 1993, pp. 109-113.

[4] D. P. Palomar, J. M. Cioffi, and M. A. Lagunas, "Joint tx-rx beamforming design for multicarrier mimo channels: a unified framework for convex optimization," IEEE Transactions on Signal Processing, vol. 51, no. 9, pp. 2381-2401, September 2003.

[5] H. H. Seung and H. L. Jae, "An overview of peak-to-average power ratio reduction techniques for multicarrier transmission," IEEE Wireless Communications, vol. 12, no. 2, pp. 56-65, April 2005.

[6] Q. H. Spencer, A. L. Swindlehurst, and M. Haardt, "Zero-forcing methods for downlink spatial multiplexing in multiuser mimo channels," Signal Processing, IEEE Transactions on [see also Acoustics, Speech, and Signal Processing, IEEE Transactions on], vol. 52, no. 2, pp. 461471, 2004.

[7] S. Serbetli and A. Yener, "Transceiver optimization for multiuser mimo systems," Signal Processing, IEEE Transactions on, vol. 52, no. 1, pp. 214-226, 2004.

[8] F. Rashid, H. Peh, and A. Manikas, "Diffused channel estimation and reception for cyclic prefix-free mc-cdma arrayed-mimo communication systems," International Journal of Wireless Information Networks, 2008.

[9] H. Ochiai and H. Imai, "On the distribution of the peak-to-average power ratio in ofdm signals," IEEE Transactions on Communications, vol. 49, no. 2, pp. 282-289, February 2001.

[10] X. Wang and A. Manikas, "Joint linear precoder-decoder optimization over mimo-cdma multipath channels," in Personal, Indoor and Mobile Radio Communications, 2007. PIMRC 2007. IEEE 18th International Symposium on, 2007, pp. 1-5. 